\title{
Small-scale averaging coarse-grains passive scalar turbulence
}

\author{
Tobias Bätge and Michael Wilczek ( \\ Max Planck Institute for Dynamics and Self-Organization, (MPI DS), Am Faßberg 17, \\ 37077 Göttingen, Germany \\ and Faculty of Physics, University of Göttingen, Friedrich-Hund-Platz 1, 37077 Göttingen, Germany
}

(Received 23 October 2020; accepted 14 May 2021; published 28 June 2021)

\begin{abstract}
Scalar fields which are subject to turbulent mixing typically feature a broad range of scales. When focusing on the large-scale dynamics, it remains a question how to effectively parametrize the small scales. Here, we address this question within the framework of a stochastic, one-dimensional passive scalar model. We show that small-scale averaging, i.e., an ensemble average over small-scale velocity fluctuations, results in an effective diffusivity reminiscent of phenomenological eddy viscosity models, while reducing the effective Reynolds number of the advecting velocity field. Based on that, we establish a filtering procedure that exactly maps second-order statistics of the fully resolved passive scalar field to the one obtained by small-scale averaging. Using fully resolved simulations, we show that small-scale averaging also captures higher-order large-scale statistics of passive scalar fields.
\end{abstract}

DOI: 10.1103/PhysRevFluids.6.064503

\section{INTRODUCTION}

Turbulent mixing plays an important role for many processes in nature and engineering [1,2] such as the dispersal of pollutants and aerosols in the atmosphere [3] or marine microorganisms in the ocean [4-7]. In such settings, large-scale quantities are often of interest, which motivates the search for effective coarse-grained descriptions. Capturing the effect of the complex multiscale dynamics of turbulent mixing on the large scales remains a computational and theoretical challenge.

To study turbulent mixing in its essence, one commonly considers passive scalar turbulence, i.e., a scalar field which is passively advected with the flow and subject to diffusion, without any back reaction on the flow [8,9]. Even in this simple setting, the resulting fields show an intermittent spatial structure with shallow ramps and steep cliffs [10-15]. Conceptually simple models such as the Kraichnan model [16], i.e., the advection of a scalar field in a white-in-time Gaussian field, have proven to be instrumental to gain insights into phenomena such as intermittency and anomalous scaling [17-19]. In particular, such models also highlight that many of the relevant phenomena do not depend on the details of the underlying flow field but are rather inherent to the mixing process itself.

In this spirit, we here consider a passive scalar advected by a compressible Kraichnan flow in one dimension. This simple model features advection and diffusion, thereby sharing characteristics of scalar turbulence. Hence, the model is suited to develop and test coarse-graining approaches. While

\footnotetext{
"michael.wilczek@ds.mpg.de
}

Published by the American Physical Society under the terms of the Creative Commons Attribution 4.0 International license. Further distribution of this work must maintain attribution to the author(s) and the published article's title, journal citation, and DOI. Open access publication funded by the Max Planck Society. 
those are not necessarily quantitatively transferable to other flows, they can provide qualitative insights.

Here, we explore to what extent the effects of spatial coarse-graining can be replaced by an ensemble average over small-scale turbulent fluctuations. We find that averaging over the small-scale velocity fluctuations effectively coarse-grains passive scalar turbulence. Through this small-scale averaging, the small scales of the passive scalar field are smoothed, while the statistics of the large scales remain largely unaffected. Within the framework of the Kraichnan model, the procedure leads to closed large-scale equations. In contrast to large eddy simulation (LES) approaches for passive scalar turbulence [20-22], the coarse-grained equations do not need to be augmented with explicit models for the unresolved scales. Yet, to establish the connection to filtering approaches, which, for example, are used for developing LES, we additionally construct a filter. The filter is designed to match the spectra of the filtered fully resolved passive scalar and the small-scale-averaged passive scalar fields, leading to identical second-order statistics. Using direct numerical simulations, we furthermore show that also higher-order statistics are very similar in the two cases.

The paper is organized as follows. We introduce the model in Sec. II. In Sec. III, we explain small-scale averaging and the filter construction. We then validate the approach by comparing the scalar energy budget and statistics of the large scales obtained by small-scale averaging and filtering a fully resolved system in Sec. IV, before we conclude in Sec. V.

\section{PASSIVE SCALAR MODEL}

The dynamics of a passive scalar field is described by an advection-diffusion equation. In the Kraichnan model, the advecting velocity field is not governed by the Navier-Stokes equations but instead is a Gaussian random field, delta correlated in time [16]. Here, we consider a onedimensional passive scalar field $\theta(x, t)$ on a periodic domain, which evolves according to

$$
\partial_{t} \theta+u \partial_{x} \theta=\kappa \partial_{x}^{2} \theta+f
$$

where $\kappa$ denotes the diffusion constant. The advecting velocity field $u(x, t)$ and the forcing $f(x, t)$ are two independent, white-in-time Gaussian random fields with spatial correlations:

$$
\begin{gathered}
\left\langle u\left(x_{1}, t_{1}\right) u\left(x_{2}, t_{2}\right)\right\rangle=Q^{u}\left(x_{1}-x_{2}\right) \delta\left(t_{1}-t_{2}\right), \\
\left\langle f\left(x_{1}, t_{1}\right) f\left(x_{2}, t_{2}\right)\right\rangle=F\left(x_{1}-x_{2}\right) \delta\left(t_{1}-t_{2}\right) .
\end{gathered}
$$

Note that, therefore, Eq. (1) is a stochastic partial differential equation in the Stratonovich sense. For the velocity field, we choose a model spectrum of the following form, inspired by [23]:

$$
E_{k} \propto k^{-5 / 3}\left(\frac{k L_{0}}{\left[\left(k L_{0}\right)^{2}+1\right]^{1 / 2}}\right)^{5 / 3+2} e^{-k l} .
$$

Here, $L_{0}$ and $l$ determine the largest and smallest length scales, respectively.

Our analytical results are complemented with simulations on a periodic domain of length $L=$ $160 \pi$ (code units). We employ a pseudospectral scheme with a spatial resolution of $N=2^{15}$. For time stepping, we use the Itô representation and an Euler-Maruyama integration scheme with a time step $\Delta t=10^{-7}$ (code units), taking into account the Itô-Stratonovich correction [24]. The large-scale forcing has unit variance and acts only on wave number $k=3 \Delta k$, where $\Delta k=2 \pi / L$. Additionally, we set the zeroth Fourier mode of the scalar field to zero. This acts as a large-scale sink and leaves the dynamics of all other modes unaffected. We impose the spectrum of the velocity field, Eq. (2), as also shown in Fig. 2. The velocity field has unit variance, and for the two length scales, we choose $L_{0}=0.05 L$ and $l \approx 2 \times 10^{-4} L$. Moreover, the diffusivity is $\kappa \approx 0.038$ (code units). Even though the velocity in our model is a random field without explicit viscosity, one can define an effective Schmidt number. Here, we interpret the cutoffs of the velocity spectrum $E_{k}$ and the scalar spectrum $C_{k}$ as the wave numbers corresponding to the Kolmogorov scale $\eta_{\mathrm{K}}$ and Batchelor scale 


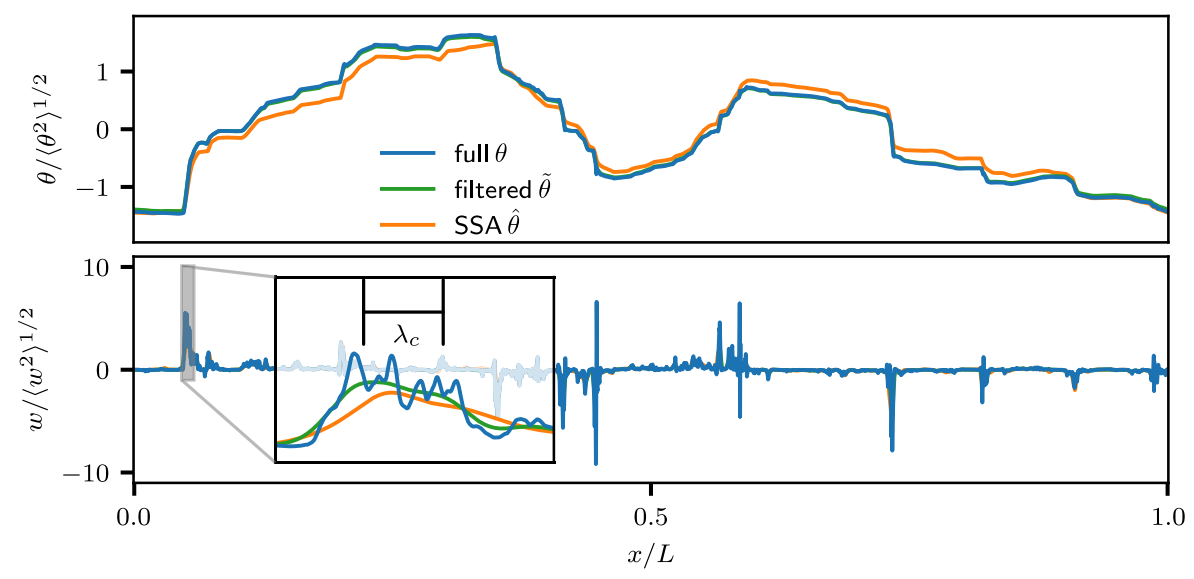

FIG. 1. Fully resolved, filtered, and small-scale-averaged scalar fields (the latter two are defined below) and corresponding gradient fields of an example realization resolved with $N=2^{15}$ grid points. The extreme events in the form of cliffs in the scalar field are represented as strong peaks in the gradient field. On the large scales, all three scalar fields share a similar structure. The zoom into one cliff structure highlights the differences between the three field types on the scale of the filter length $\lambda_{c}$.

$\eta_{\mathrm{B}}$, respectively:

$$
\frac{8}{5} \frac{\sum k^{6} E_{k} \Delta k}{\sum k^{4} E_{k} \Delta k} \approx k_{\eta_{\mathrm{K}}}^{2}, \quad \frac{14}{8} \frac{\sum k^{6} C_{k} \Delta k}{\sum k^{4} C_{k} \Delta k} \approx k_{\eta_{\mathrm{B}}}^{2} .
$$

The sums are dominated by different powers of the respective cutoff wave numbers and, therefore, allow an estimation of those. The different prefactors originate from the scaling of the two spectra, i.e., $-\frac{5}{3}$ for the velocity spectrum and $-\frac{7}{3}$ for the scalar spectrum. A Schmidt number can be defined through the ratio of Kolmogorov and Batchelor scales [11]:

$$
\mathrm{Sc} \equiv\left(\frac{\eta_{\mathrm{K}}}{\eta_{\mathrm{B}}}\right)^{2}=\left(\frac{k_{\eta_{\mathrm{B}}}}{k_{\eta_{\mathrm{K}}}}\right)^{2} \approx 0.3 .
$$

We also define a Peclet number Pe $\equiv Q^{u}(0) / \kappa \approx 26$ and a Reynolds number for the velocity field via the scale separation:

$$
\operatorname{Re} \equiv\left(\frac{L_{0}}{\eta_{\mathrm{K}}}\right)^{4 / 3} \approx 1000
$$

An example realization of scalar and gradient $\left(w=\partial_{x} \theta\right)$ fields, is shown in Fig. 1. As typical for scalar turbulence, the scalar field features cliffs $[9,11,14,25,26]$, which are the smallest structures and correspond to strong peaks and extreme events in the gradient field.

\section{COARSE-GRAINED DYNAMICS}

\section{A. Common filter approach}

Filtering is a versatile tool for analyzing turbulent flows to gain insights into the interaction between different length scales and structures of turbulence [27-30]. Furthermore, it is the foundation for approaches aiming at coarse-grained dynamics such as LES [23]. To obtain such large-scale dynamics, one usually applies a low-pass filter. In real space, filtering is defined by a convolution integral with the filter kernel $G$, which becomes a product of the respective Fourier components in 
Fourier space:

$$
\tilde{\theta}(x)=\int d y G(x-y) \theta(y), \quad \tilde{\theta}_{k}=G_{k} \theta_{k} .
$$

Here, the subscript $k$ denotes the Fourier mode corresponding to the wave number $k$ (assuming periodic boundary conditions). Applying the filter to the equation of motion, we obtain the exact large-scale equation

$$
\partial_{t} \tilde{\theta}+\widetilde{u \partial_{x}} \theta=\kappa \partial_{x}^{2} \tilde{\theta}+\tilde{f}
$$

The filtered dynamics, however, are not closed as the advection contribution involves the filtered product of two fields. In the framework of large eddy simulations, this filtered product is expressed as a product of the filtered fields, and the residual terms are modeled (see, e.g., [21]).

\section{B. Small-scale averaging}

Low-pass filtering is essentially a local weighted volume average over small scales while large scales remain unchanged. Therefore, it seems plausible to average directly over small-scale fluctuations to obtain coarse-grained dynamics without filtering. For the model discussed here, we propose a coarse-graining by an ensemble average over the small-scale velocity fluctuations rather than scalar fluctuations or both. We refer to this procedure as small-scale averaging (SSA). As we will show in the following, in the framework of the Kraichnan model, this results in a closed effective large-scale equation with an effective diffusivity reminiscent of eddy viscosity models. Hence, there is no need for modeling unclosed terms. Intuitively, small-scale velocity fluctuations distort the scalar field on the corresponding length scales, while leaving the large scales unaffected. As a result, small-scale averaging captures the large scales while the small scales are smoothed out.

To derive the effective large-scale equation, we split the velocity field into large scales $U$ and fluctuations $u^{\prime}$ with a sharp Fourier filter, as visualized in Fig. 2:

$$
u(x, t)=\sum_{k} u_{k}(t) e^{i k x}=\underbrace{\sum_{|k|<k_{c}} u_{k}(t) e^{i k x}}_{U(x, t)}+\underbrace{\sum_{|k| \geqslant k_{c}} u_{k}(t) e^{i k x}}_{u^{\prime}(x, t)} .
$$

Next, we introduce an ensemble average over the small-scale fluctuations $u^{\prime}$, and denote $\hat{\theta}=\langle\theta\rangle_{u^{\prime}}$ as the small-scale-averaged scalar field. The wave number separating fluctuations and large scales, $k_{c}$, defines the coarse-graining length scale $\lambda_{c}=2 \pi / k_{c}$. The forcing $f$ and the large-scale velocity field $U$ are statistically independent of the small-scale fluctuations $u^{\prime}$. Hence, they commute with an ensemble average over realizations of $u^{\prime}$. Applying this average then to the equation of motion, Eq. (1), results in

$$
\partial_{t} \hat{\theta}+U \partial_{x} \hat{\theta}=-\left\langle u^{\prime} \partial_{x} \theta\right\rangle_{u^{\prime}}+\kappa \partial_{x}^{2} \hat{\theta}+f .
$$

Since the velocity fluctuations are a Gaussian random field, one can evaluate the average advection term $\left\langle u^{\prime} \partial_{x} \theta\right\rangle_{u^{\prime}}=-\frac{1}{2} Q^{u^{\prime}}(0) \partial_{x}^{2} \hat{\theta}$ using the Furutsu-Donsker-Novikov identity [31-33]. Here, $Q^{u^{\prime}}(0)$ is the spatial correlation function of $u^{\prime}$ evaluated at the origin. This leads to an additional diffusive term proportional to the energy contained in the fluctuations. Additionally, the small scales of the advecting velocity field are removed, which implies an effectively reduced Reynolds number $\operatorname{Re}^{\prime}=$ $\left(\eta_{\mathrm{K}} / \lambda_{c}\right)^{4 / 3} \operatorname{Re}$ [cf. Eq. (5)]. For our concrete numerical example (see Fig. 2), this leads to $\operatorname{Re}^{\prime} \approx 12$. Thus, our proposed effective large-scale equation has a closed and exact form:

$$
\partial_{t} \hat{\theta}+U \partial_{x} \hat{\theta}=\underbrace{\left[\kappa+\frac{1}{2} Q^{u^{\prime}}(0)\right]}_{\kappa_{\text {eff }}} \partial_{x}^{2} \hat{\theta}+f .
$$

Despite the complementary approach, the effective diffusion with constant $\kappa_{\text {eff }}$ is similar to the eddydiffusivity closures for the Kraichnan model [21]. The small-scale-averaged equation of motion, 
(a)

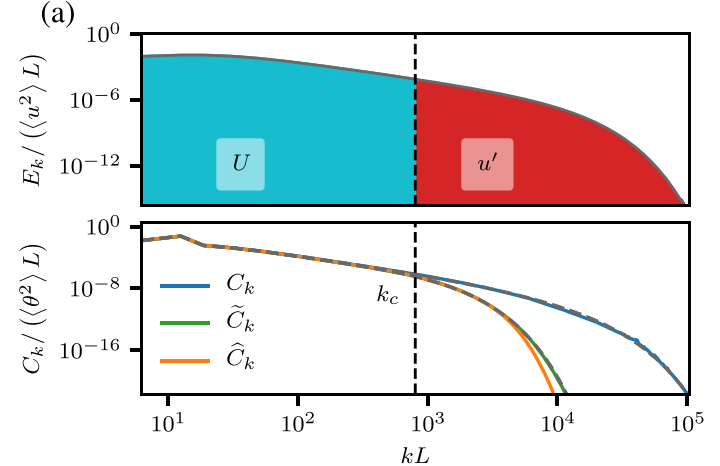

(b)

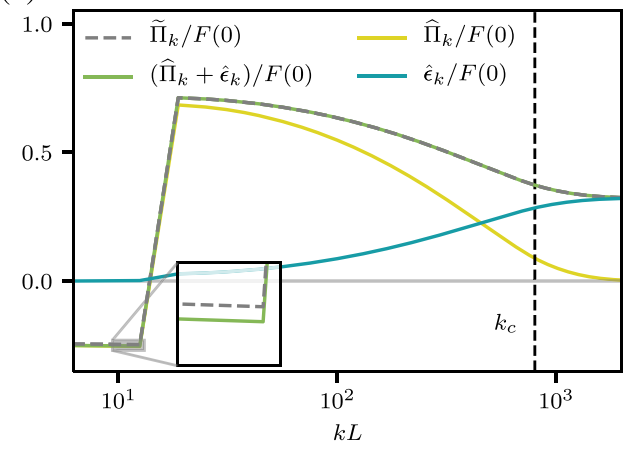

FIG. 2. Spectral characteristics of the fully resolved passive scalar system, its filtered version, and the small-scale-averaged version. (a) The top panel shows the spectrum of the advecting velocity field $u$ and illustrates the splitting into small-scale and large-scale components, $u^{\prime}$ and $U$, respectively. Here, $k_{c}=128 \Delta k$ denotes the wave number separating small and large scales, which in the following also defines the filter length scale. In the bottom panel, the fully resolved scalar spectrum $C_{k}$, filtered scalar spectrum $\widetilde{C}_{k}$, and small-scale-averaged scalar spectrum $\widehat{C}_{k}$ obtained from a simulation are compared to the respective analytical solutions (dashed gray lines) [see Eqs. (18)-(20)]. For wave numbers larger than the filter wave number, the small-scale-averaged and filtered spectrum drop off relative to the fully resolved one with minor deviations among each other at the end of the spectral cutoff. (b) Individual contributions of the balance between the small-scale-averaged and filtered spectral energy budget, Eq. (26), normalized by the forcing amplitude $F(0)$. Overall, the sum of the small-scale-averaged spectral energy flux $\widehat{\Pi}_{k}$ and the small-scale-averaged spectral energy dissipation $\hat{\epsilon}_{k}$ add up to the spectral energy flux of the filtered passive scalar, showing that SSA can capture the spectral characteristics of the filtered spectral energy budget. The minor deviation in the balance of small-scale-averaged flux and dissipation to the filtered flux contribution, Eq. (26), is highlighted with an inset; it can be traced back to the fact that our filter is not a perfect low-pass filter. It commutes with the large-scale forcing only approximately.

Eq. (10), has the same structure as the one for the full scalar, Eq. (1), i.e., it features advection by a Gaussian random field, diffusion with a constant diffusivity, and identical forcing.

For small-scale averaging being effective, the smallest scales of the velocity field should be smaller or equal to those of the scalar, i.e., $\mathrm{Sc} \leqslant 1$. Otherwise, there exists a range of scales for the scalar field in which no velocity fluctuations are present and the additional diffusion contribution in Eq. (10) may become negligible. This appears consistent with recent findings based on direct numerical simulations of three-dimensional scalar turbulence which show that turbulent mixing becomes ineffective for larger Schmidt numbers [34].

As a side note, we mention that the SSA approach can be generalized to the $d$-dimensional Kraichnan model:

$$
\partial_{t} \theta+\boldsymbol{u} \cdot \nabla \theta=\kappa \Delta \theta+f,
$$

where the scalar field $\theta(\boldsymbol{x}, t)$ is advected by an incompressible $d$-dimensional Kraichnan velocity field $\boldsymbol{u}(\boldsymbol{x}, t)$. Analogous to Eq. (8), we can split the velocity field and average over the fluctuations $\boldsymbol{u}^{\prime}$ resulting in the small-scale-averaged dynamics

$$
\begin{aligned}
\partial_{t} \hat{\theta}+\boldsymbol{U} \cdot \nabla \hat{\theta} & =-\left\langle\boldsymbol{u}^{\prime} \cdot \nabla \theta\right\rangle_{\boldsymbol{u}^{\prime}}+\kappa \Delta \hat{\theta}+f \\
& =\left(\frac{\operatorname{Tr}\left(\mathbf{Q}^{\boldsymbol{u}^{\prime}}\right)(\mathbf{0})}{2 d}+\kappa\right) \Delta \hat{\theta}+f .
\end{aligned}
$$

Similar to Eq. (10), one obtains an additional diffusivity in the form of the trace of the correlation tensor of the small-scale velocity fluctuations. 


\section{Extracting large scales from fully resolved passive scalar turbulence}

Next, we establish a connection between the traditional filtering approach and SSA, and show that small-scale averaging captures the large scales of a fully resolved system. To this end, we introduce a low-pass filter which extracts large scales from a fully resolved system. The filter is designed such that the second-order statistics of the filtered fields correspond exactly to those obtained from small-scale averaging.

Filtering modulates the amplitude of the individual Fourier components and accordingly the scalar spectrum $C_{k}=2\left\langle\theta_{k} \theta_{-k}\right\rangle / \Delta k$. Therefore, an optimal filter can be constructed by demanding that the spectrum of the filtered field coincides with that obtained from the small-scale-averaged dynamics:

$$
\widetilde{C}_{k}=G_{k}^{2} C_{k}=\widehat{C}_{k},
$$

which implies

$$
G_{k} \equiv \sqrt{\frac{\widehat{C}_{k}}{C_{k}}} .
$$

Hence, constructing the filter requires knowledge of the respective spectra. For the one-dimensional Kraichnan model, an analytical expression for the two-point correlator of the scalar field has been derived in Ref. [35], from which the spectrum can be obtained. Using the Furutsu-DonskerNovikov identity, one can derive the time evolution of the two-point correlators of the scalar $\theta$ and scalar gradient $w=\partial_{x} \theta, C(r, t)=\langle\theta(x, t) \theta(x+r, t)\rangle$ and $\Omega(r, t)=\langle w(x, t) w(x+r, t)\rangle$, respectively $[35,36]$ :

$$
\begin{gathered}
\partial_{t} C(r, t)=\left[S^{u}(r)+2 \kappa\right] \partial_{r}^{2} C(r, t)+F(r), \\
\partial_{t} \Omega(r, t)=\partial_{r}^{2}\left[\left(S^{u}(r)+2 \kappa\right) \Omega(r, t)\right]-\partial_{r}^{2} F(r) .
\end{gathered}
$$

Here, $S^{u}(r)$ corresponds to the second-order structure function of the velocity field, which can be defined as

$$
S^{u}(r)=Q^{u}(0)-Q^{u}(r) .
$$

Assuming statistical stationarity, the solution for the gradient correlator has been explicitly obtained in Ref. [35] and takes the form

$$
\Omega(r)=\frac{F(r)}{2 \kappa+S^{u}(r)}-\frac{A}{2 \kappa+S^{u}(r)} .
$$

Here, $A$ is an integration constant fixed by the condition $\int_{0}^{L} d r \Omega(r)=0$, which is a consequence of the periodic boundary condition. The spectrum of the gradient field is given by a Fourier transform of the two-point correlator

$$
\Omega_{k}=\frac{1}{\pi} \int_{0}^{L} d r e^{-i k r} \Omega(r) .
$$

The scalar spectrum is then obtained by spatial integration, which is equivalent to a multiplication with $k^{-2}$ in Fourier space:

$$
C_{k}=\frac{1}{k^{2}} \Omega_{k}, \quad k \neq 0 .
$$

Because the SSA dynamics is identical to that of the full field, the corresponding SSA correlators and spectra, as well as their evolution equations, have an identical functional form. The evolution equations for the SSA correlators can be obtained from (15) and (16) simply by replacing the second-order structure function of the full velocity field by that of the large-scale field, and by 
replacing the diffusivity with the effective diffusivity. This leads to the corresponding small-scaleaveraged equations:

$$
\begin{gathered}
\partial_{t} \widehat{C}(r, t)=\left[S^{U}(r)+2 \kappa_{\mathrm{eff}}\right] \partial_{r}^{2} \widehat{C}(r, t)+F(r), \\
\partial_{t} \widehat{\Omega}(r, t)=\partial_{r}^{2}\left[\left(S^{U}(r)+2 \kappa_{\mathrm{eff}}\right) \widehat{\Omega}(r, t)\right]-\partial_{r}^{2} F(r),
\end{gathered}
$$

where $S^{U}$ denotes the second-order structure function of the large-scale velocity field $U$. Based on this, the correlator of the SSA gradient field takes the form analogous to (18), and through (19) and (20) we can compute the spectrum $\widehat{C}_{k}$ of the SSA scalar field. In Fig. 2, the spectra resulting from the analytical computations in this section are compared to spectra obtained from simulations of the fully resolved and small-scale-averaged dynamics, Eqs. (1) and (10), respectively, demonstrating good agreement. Figure 2 also shows that for wave numbers larger than $k_{c}$ the SSA spectrum drops off in comparison to the fully resolved one, showing that $k_{c}$ signifies the wave number beyond which the smoothing effect of SSA sets in. With the results on the spectra of the fully resolved and the SSA scalar fields, we can explicitly express the filter kernel according to Eq. (14). When applied to a fully resolved scalar field, such a filter leads to a coarser scalar field with less extreme gradients as shown in Fig. 1.

\section{COMPARISON OF FULLY RESOLVED, FILTERED, AND SSA SIMULATIONS}

\section{A. Spectral scalar energy budget}

The spectral scalar energy budget characterizes the time evolution of the scalar spectrum. We now show that small-scale averaging essentially has the effect of filtering on the fully resolved scalar energy budget. As the spectrum is the Fourier transform of the corresponding two-point correlator, we obtain the evolution equation for the SSA spectrum from Eq. (21):

$$
\partial_{t} \widehat{C}_{k}=\widehat{T}_{k}-2 \kappa_{\mathrm{eff}} k^{2} \widehat{C}_{k}+F_{k}, \quad \text { with } \quad \widehat{T}_{k}=\mathcal{F}\left\{S^{U}(r) \partial_{r}^{2} \widehat{C}(r, t)\right\}_{k},
$$

where $\widehat{T}_{k}$ denotes the small-scale-averaged scalar transfer, and $F_{k}$ denotes the forcing spectrum. In comparison, the spectral budget of the filtered field takes the form

$$
\partial_{t} \widetilde{C}_{k}=G_{k}^{2} T_{k}-2 \kappa k^{2} \widetilde{C}_{k}+\widetilde{F}_{k}, \quad \text { with } \quad T_{k}=\mathcal{F}\left\{S^{u}(r) \partial_{r}^{2} C(r, t)\right\}_{k} .
$$

Here, the tilde denotes quantities based on the filtered scalar dynamics, and $T_{k}$ is the unfiltered scalar transfer.

In a statistically stationary state $\widetilde{C}_{k}=\widehat{C}_{k}$ holds due to our filter choice defined by Eq. (14). Also, the filter is a low-pass filter, which approximately leaves the spectrum of the large-scale forcing unaffected, i.e., $\widetilde{F}_{k}=G_{k}^{2} F_{k} \approx F_{k}$. We also test the validity of this approximation numerically in terms of the energy budget (see inset of Fig. 2). Using those two properties, we compare the different contributions to the scalar energy budget.

Subtracting (24) from (23) and assuming a statistically stationary state, only the transfer terms and one diffusive term originating from the difference between $\kappa$ and $\kappa_{\text {eff }}=\kappa+\frac{1}{2} Q^{u^{\prime}}(0)$ remain. We thereby obtain a relation between filtered and the small-scale-averaged scalar transfer

$$
G_{k}^{2} T_{k} \approx \widehat{T}_{k}-Q^{u^{\prime}}(0) k^{2} \widehat{C}_{k}
$$

This shows that the filtered scalar transfer is related to a combination of the SSA scalar transfer and the additional diffusivity which arises from the averaged small-scale velocity field. To further compare the individual contributions, we sum this equation up to wave number $k$ and obtain

$$
\widetilde{\Pi}_{k} \approx \widehat{\Pi}_{k}+\hat{\epsilon}_{k} .
$$



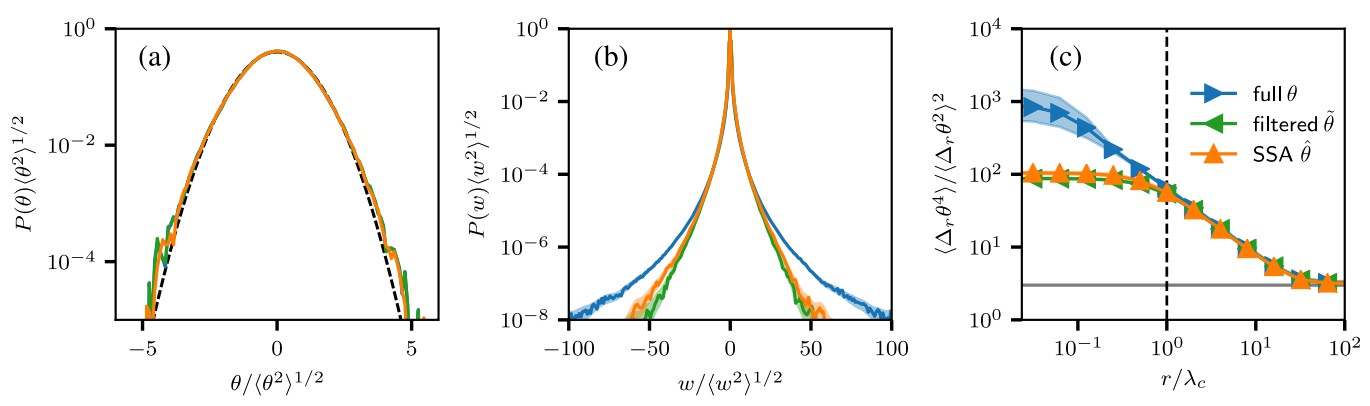

FIG. 3. Statistics of the fully resolved, filtered, and small-scale-averaged scalar fields. (a) All three onepoint scalar PDFs are close to Gaussian, here marked by a black dashed line. Note that the PDFs of the full and the filtered fields are almost indistinguishable. (b) The gradient PDFs show the characteristic heavy tails and a good agreement between filtered and small-scale-averaged PDFs up to the outer tails, which are significantly less heavy than those of the fully resolved, unfiltered fields. The transparent areas correspond to confidence intervals of 95\% obtained by a basic bootstrap [37]. (c) For increments larger than the filter length, all three scalar fields show a similar flatness, approaching the Gaussian value for large increments (gray line). Here, the transparent areas also correspond to the confidence intervals of $95 \%$.

Here,

$$
\widetilde{\Pi}_{k}=-\sum_{k^{\prime}=0}^{k} G_{k^{\prime}}^{2} T_{k^{\prime}} \Delta k, \quad \widehat{\Pi}_{k}=-\sum_{k^{\prime}=0}^{k} \widehat{T}_{k^{\prime}} \Delta k, \quad \text { and } \quad \hat{\epsilon}_{k}=\sum_{k^{\prime}=0}^{k} Q^{u^{\prime}}(0) k^{\prime 2} \widehat{C}_{k^{\prime}} \Delta k
$$

are the scalar flux terms and the scalar dissipation up to wave number $k$ by the additional diffusivity due to small-scale averaging. To visually show the validity of the spectral balance, Eq. (26), all contributions are compared individually in Fig. 2. Across all scales, the balance is satisfied. This demonstrates that small-scale averaging captures the effect filtering has on the fully resolved scalar flux and energy budget. That means, given an appropriately defined filter, the second-order statistics of the coarse-grained scalar field are identical to those obtained by small-scale averaging.

\section{B. Comparison of higher-order statistics}

Through simulations, we additionally verify that our approach also captures higher-order statistics and spatial structure. We expect the fully resolved, filtered, and small-scale-averaged scalar fields to have similar one-point statistics since all three feature a very similar large-scale structure as illustrated in Fig. 1. Indeed, the three scalar one-point probability density functions (PDFs) shown in Fig. 3 are close to Gaussian and match very well.

The gradient, which is naturally more sensitive to small-scale features, is strongly affected by filtering and small-scale averaging. Because of the shallower cliff structures in the SSA and filtered fields, the corresponding gradient PDFs have less heavy tails than the fully resolved PDF. Importantly, the gradient PDFs of the filtered and small-scale-averaged fields agree up to the outer tails, which shows that small-scale-averaged fields feature higher-order one-point statistics similar to the filtered fields.

Regarding two-point statistics, we turn to the flatness $\left\langle\Delta_{r} \theta(x)^{4}\right\rangle /\left\langle\Delta_{r} \theta(x)^{2}\right\rangle^{2}$ of scalar increments, $\Delta_{r} \theta(x)=\theta(x+r)-\theta(x)$. For very small spatial separation, we essentially sample the gradient field. Corresponding to the PDFs discussed previously, the fully resolved scalar field has an increased flatness for the small increments compared to the SSA and filtered fields. Throughout the range of separations, the small-scale-averaged and filtered scalar have a very similar increment flatness. Moreover, for separations larger than the filter length, all three fields have similar flatnesses and finally approach the Gaussian value 3 for very large separations. This indicates that small-scale averaging reproduces the spatial structure of the large scales from fully resolved simulations. 


\section{CONCLUSION}

We introduced the SSA approach for obtaining an effective large-scale equation for passive scalar turbulence. Within the framework of a one-dimensional Kraichnan model for passive scalar turbulence, we have shown that an ensemble average over the small-scale fluctuations of the advecting velocity field leads to closed equations for the coarse-grained dynamics. This procedure leads to an additional diffusivity of the scalar field due to small-scale velocity fluctuations, while effectively reducing the Reynolds number of the advecting velocity field.

To enable quantitative comparisons to the large-scale statistics of fully resolved fields, we have established a filter that maps the large scales extracted from fully resolved simulations to those obtained by our approach. Based on this, we compared the energy budgets of the full, filtered, and SSA fields, finding that the SSA approach indeed captures second-order large-scale statistics. We also compared higher-order statistics by means of fully resolved simulations, finding a good agreement between the statistics of the filtered fields and the SSA fields.

As briefly discussed, this procedure can also be applied to the Kraichnan model in any dimension. Furthermore, it could also be interesting to explore to what extent the presented approach can be transferred to a passive scalar advected by a turbulent flow governed by the Navier-Stokes equations, which lacks the idealized features of the Kraichnan model. In real turbulence, the advecting velocity field is non-Gaussian and continuous in time, making an exact analytical approach infeasible. Motivated by the fact that SSA and filtering effectively reduce the Reynolds number of the advecting velocity field while introducing a corresponding turbulent diffusion of the scalar field, the investigation of a systematic mapping of the large-scale statistics of passive scalar turbulence at various Reynolds and Schmidt numbers could be an interesting direction for further work.

\section{ACKNOWLEDGMENTS}

We thank Gerrit Green for helpful discussions. This work was supported by the Max Planck Society and the Priority Programme SPP 1881 Turbulent Superstructures of the Deutsche Forschungsgemeinschaft.

[1] S. I. Abarzhi, S. Gauthier, and K. R. Sreenivasan, Turbulent mixing and beyond: Non-equilibrium processes from atomistic to astrophysical scales I, Philos. Trans. R. Soc. A 371, 20120436 (2012).

[2] S. I. Abarzhi, S. Gauthier, and K. R. Sreenivasan, Turbulent mixing and beyond: Non-equilibrium processes from atomistic to astrophysical scales II, Philos. Trans. R. Soc. A 371, 20130268 (2013).

[3] J. M. Stockie, The mathematics of atmospheric dispersion modeling, SIAM Rev. 53, 349 (2011).

[4] M. Niazi Ardekani, G. Sardina, L. Brandt, L. Karp-Boss, R. N. Bearon, and E. A. Variano, Sedimentation of inertia-less prolate spheroids in homogenous isotropic turbulence with application to non-motile phytoplankton, J. Fluid Mech. 831, 655 (2017).

[5] M. Borgnino, G. Boffetta, F. De Lillo, and M. Cencini, Gyrotactic swimmers in turbulence: Shape effects and role of the large-scale flow, J. Fluid Mech. 856, R1 (2018).

[6] W. M. Durham, E. Climent, M. Barry, F. De Lillo, G. Boffetta, M. Cencini, and R. Stocker, Turbulence drives microscale patches of motile phytoplankton, Nat. Commun. 4, 2148 (2013).

[7] R. E. Breier, C. C. Lalescu, D. Waas, M. Wilczek, and M. G. Mazza, Emergence of phytoplankton patchiness at small scales in mild turbulence, Proc. Natl. Acad. Sci. USA 115, 12112 (2018).

[8] B. I. Shraiman and E. D. Siggia, Scalar turbulence, Nature (London) 405, 639 (2000).

[9] K. R. Sreenivasan, Turbulent mixing: A perspective, Proc. Natl. Acad. Sci. USA 116, 18175 (2018).

[10] K. R. Sreenivasan, R. A. Antonia, and D. Britz, Local isotropy and large structures in a heated turbulent jet, J. Fluid Mech. 94, 745 (1979).

[11] M. Holzer and E. D. Siggia, Turbulent mixing of a passive scalar, Phys. Fluids 6, 1820 (1994). 
[12] A. Celani, A. Lanotte, A. Mazzino, and M. Vergassola, Fronts in passive scalar turbulence, Phys. Fluids 13, 1768 (2001).

[13] T. Watanabe and T. Gotoh, Intermittency in passive scalar turbulence under the uniform mean scalar gradient, Phys. Fluids 18, 058105 (2006).

[14] K. P. Iyer, J. Schumacher, K. R. Sreenivasan, and P. K. Yeung, Steep Cliffs and Saturated Exponents in Three-Dimensional Scalar Turbulence, Phys. Rev. Lett. 121, 264501 (2018).

[15] D. Buaria, M. P. Clay, K. R. Sreenivasan, and P. K. Yeung, Small-Scale Isotropy and Ramp-Cliff Structures in Scalar Turbulence, Phys. Rev. Lett. 126, 034504 (2021).

[16] R. H. Kraichnan, Small-scale structure of a scalar field convected by turbulence, Phys. Fluids 11, 945 (1968).

[17] R. H. Kraichnan, Anomalous Scaling of a Randomly Advected Passive Scalar, Phys. Rev. Lett. 72, 1016 (1994).

[18] G. Falkovich, K. Gawȩdzki, and M. Vergassola, Particles and fields in fluid turbulence, Rev. Mod. Phys. 73, 913 (2001).

[19] J. Cardy, G. Falkovich, and K. Gawedzki, Non-Equilibrium Statistical Mechanics and Turbulence, edited by S. Nazarenko and O. V. Zaboronski (Cambridge University Press, Cambridge, 2008).

[20] M. M. Afonso, A. Celani, R. Festa, and A. Mazzino, Large-eddy-simulation closures of passive scalar turbulence: A systematic approach, J. Fluid Mech. 496, 355 (2003).

[21] A. Celani, M. M. Afonso, and A. Mazzino, Coarse-grained description of a passive scalar, J. Turbul. 7, N52 (2006).

[22] S. Hickel, N. A. Adams, and N. N. Mansour, Implicit subgrid-scale modeling for large-eddy simulation of passive-scalar mixing, Phys. Fluids 19, 095102 (2007).

[23] S. B. Pope, Turbulent Flows (Cambridge University Press, Cambridge, 2000).

[24] P. E. Kloeden and E. Platen, Numerical Solution of Stochastic Differential Equations (Springer, Berlin, Heidelberg, 1992).

[25] S. Chen and R. H. Kraichnan, Simulations of a randomly advected passive scalar field, Phys. Fluids 10, 2867 (1998).

[26] K. P. Iyer, J. Schumacher, K. R. Sreenivasan, and P. K. Yeung, Fractal iso-level sets in high-Reynoldsnumber scalar turbulence, Phys. Rev. Fluids 5, 044501 (2020).

[27] M. Germano, Turbulence: The filtering approach, J. Fluid Mech. 238, 325 (1992).

[28] H. Aluie and G. L. Eyink, Localness of energy cascade in hydrodynamic turbulence. II. Sharp spectral filter, Phys. Fluids 21, 115108 (2009).

[29] G. L. Eyink and H. Aluie, Localness of energy cascade in hydrodynamic turbulence. I. Smooth coarse graining, Phys. Fluids 21, 115107 (2009).

[30] T. D. Drivas, P. L. Johnson, C. C. Lalescu, and M. Wilczek, Large-scale sweeping of small-scale eddies in turbulence: A filtering approach, Phys. Rev. Fluids 2, 104603 (2017).

[31] K. Furutsu, On the statistical theory of electromagnetic waves in a fluctuating medium (I), J. Res. Natl. Bur. Stand. (U. S.) 67D, 303 (1963).

[32] M. D. Donsker, On function space integrals, in Proceedings Conference on Theory and Application of Analysis in Function Space (MIT Press, Cambridge, 1964), pp. 17-30 [Matematika 11, 128 (1967)].

[33] E. A. Novikov, Functionals and the random force method in turbulence theory, Sov. Phys. JETP 20, 1290 (1965).

[34] D. Buaria, M. P. Clay, K. R. Sreenivasan, and P. K. Yeung, Turbulence is an Ineffective Mixer When Schmidt Numbers are Large, Phys. Rev. Lett. 126, 074501 (2021).

[35] M. Chertkov, I. Kolokolov, and M. Vergassola, Inverse cascade and intermittency of passive scalar in one-dimensional smooth flow, Phys. Rev. E 56, 5483 (1997).

[36] M. Vergassola and A. Mazzino, Structures and Intermittency in a Passive Scalar Model, Phys. Rev. Lett. 79, 1849 (1997).

[37] A. C. Davison and D. V. Hinkley, Bootstrap Methods and their Application, Cambridge Series in Statistical and Probabilistic Mathematics (Cambridge University Press, Cambridge, UK, 1997). 